\title{
Nonminimum-Phase Compensation for Nonlinear Processes
}

\author{
Raymond A. Wright and Costas Kravaris \\ Dept. of Chemical Engineering, University of Michigan, Ann Arbor, MI 48109
}

\begin{abstract}
The design of controllers for nonlinear, nonminimum-phase processes is one of the most difficult control problems currently faced. Current available control algorithms for nonlinear processes rely implicitly or explicitly on an inverse of the process. Linear control methods for nonminimum-phase processes are based on a decomposition of the process into a minimum-phase and a nonminimum-phase part. Such a decomposition is an open problem for nonlinear systems.

In this work, a control structure called the minimum-phase output predictor for nonlinear, nonminimum-phase processes is developed. The structure is based on the notion of statically equivalent outputs; a minimum-phase, statically equivalent output is estimated on-line and then an available nonlinear control algorithm is used to control it to set point. The advantage of the proposed formulation is that it is based on the calculation of an output function, not on a decomposition of the process dynamics. The proposed control methodology is applied and its performance is evaluated for a chemical engineering example.
\end{abstract}

\section{Introduction}

The problem of constructing control algorithms for processes with unstable inverses is a key issue in nonlinear process control, since the available control algorithms rely either implicitly (Kravaris and Chung, 1987) or explicitly (Economou et al., 1986) on generating an inverse of the process. Methods are available for linear systems with unstable inverses, which are modeled with a transfer function. They are all based on a factorization of the process transfer function, $G_{p}(s)$, of the form $G_{p}(s)=G_{p+}(s) \cdot G_{p-}(s)$ where $G_{p-}(s)$ is minimum phase and $G_{p+}(0)=1$. Available methods include the internal model control of Garcia and Morari (1982) and the generalized Smith predictor (Ramanathan et al., 1989). Under the assumption of a perfect model, these approaches are equivalent to controlling the corresponding minimum-phase system, leaving the nonminimum-phase component in open loop. One assumption is central in all predictor-type approaches and will also be necessary in this work. The system under consideration is assumed to be open-loop stable. The general structures lead to a control law that preserves the stability of the system and regulates the output to set point. In addition to these requirements, it is also desirable to select the controller so that it also optimizes

R. A. Wright is presently at The Dow Chemical Company, 1400 Building, Midland, MI 48667 . a certain performance criterion, such as the integral square error (ISE). The factorization given above, with $G_{p+}(s)$ being an all-pass, for internal model control or the generalized Smith predictor has been shown to lead to ISE-optimal control with respect to step changes.

For nonlinear systems, results on decomposition into minimum-phase and nonminimum-phase subsystems are available only for second-order systems in Kravaris and Daoutidis (1990), who also developed ISE-optimal decomposition and derived static state feedback synthesis formulas. For general nonlinear systems, decomposition of the dynamics into a minimum-phase and a nonminimum-phase part is an open problem. This work will introduce the notion of statically equivalent outputs; this notion will be used to develop an output feedback control structure called the minimum-phase output predictor to estimate a statically equivalent, minimum-phase output on-line and to control it to set point. The control of the staticallyequivalent, minimum-phase output is based on the input/output linearization method of Kravaris and Chung (1987). The advantage of the proposed formulation is that it is based on the calculation of an output function and not on a decomposition of the dynamics, as is typically done in linear control methods. The issue of optimal choice of statically equivalent, minimum-phase output is also addressed in this article in terms 
of the ISE criterion. Results that characterize the ISE-optimal output are obtained as well as specific formulas in special cases.

In the first section, the generalized Smith predictor for linear systems is reviewed in transfer function form. The second section develops a state space version using an on-line estimation of an output function, instead of a decomposition. The notion of statically equivalent outputs is then defined. The minimum-phase output predictor for nonlinear systems is then developed in terms of an arbitrary, statically equivalent, minimum-phase output. The following section gives rigorous mathematical results on the ISE-optimization problem for the class of nonlinear systems that can be described in natural coordinates. In particular, the notion of ISE-optimal output is defined, and necessary conditions for ISE-optimality are derived. The final section illustrates the application of the proposed control methodology and evaluates its performance in a chemical engineering example.

\section{Generalized Smith Predictor for Linear Processes}

Consider a SISO open-loop stable linear process with transfer function $G_{p}(s) . G_{p}(s)$ can then be factored into $G_{p}(s)=G_{p+}(s) \cdot G_{p-}(s)$, where $G_{p-}(s)$ is stable and minimum phase and $G_{p+}(0)=1$. The generalized Smith predictor (GSP) (Ramanathan et al., 1989) simulates the difference between the process transfer function with and without the nonminimum-phase factor. The corrective signal, $\delta y$, is added to the measured output to predict what the output would have been if there were no nonminimum-phase factor present. This prediction, $y^{*}$, is fed to the controller $G_{c}(s)$. The GSP structure is shown in Figure 1a.

A straightforward calculation gives the closed-loop transfer function:

$$
\frac{y(s)}{y_{s p}(s)}=\frac{G_{c}(s) G_{p-}(s)}{1+G_{c}(s) G_{p-}(s)} G_{p+}(s)
$$

The form of the closed-loop transfer function and the interpretation of the feedback signal $y^{*}$ indicate that, under the assumption of a perfect model, the GSP scheme is equivalent to controlling the corresponding minimum-phase system,

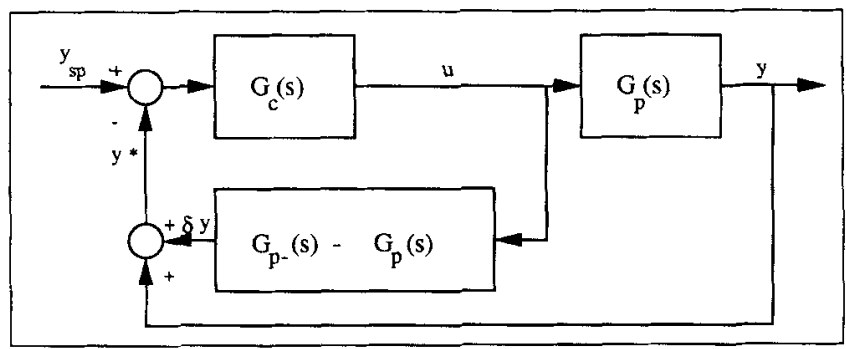

Figure 1a. Generalized Smith predictor structure.

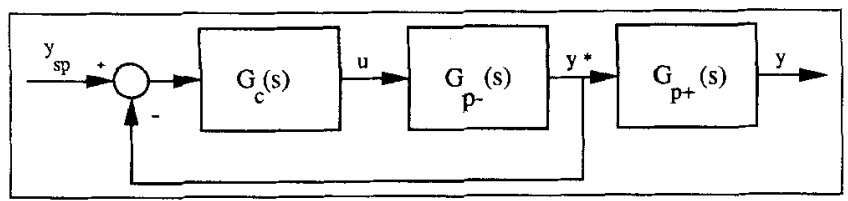

Figure 1b. Equivalent structure for the generalized Smith predictor.
$G_{p-}(s)$, as shown in Figure $1 \mathrm{~b}$, while $G_{p+}(s)$ is left in open loop. Thus, the controller, $G_{c}(s)$, will be parameterized on the basis of the minimum-phase part of the model via a standard synthesis formula. It is important to observe here that the properties of the factorization of $G_{p}(s)$ imply the following very important properties for the corrected feedback signal $y^{*}$ :

- $y^{*}$ goes to $y_{s p}$ if, and only if, $y$ goes to $y_{s p}$.

- The transfer function between $u$ and $y^{*}$ is minimum phase.

The given factorization is clearly not unique. The particular factorization, where $G_{p+}(s)$ is chosen to be an all-pass, results in an ISE-optimal response of $y$ for step changes in $y_{s p}$ in the limit as perfect control of $y^{*}$ to set point is achieved (Newton et al., 1957).

\section{State-Space Calculation and Interpretation of the Minimum-Phase Output for Linear Systems}

In this section, $y^{*}$ will be given a state space interpretation for linear systems as an auxiliary output, which has the same static gain as $y$ and makes the system minimum phase. This interpretation will naturally carry over to nonlinear systems. Additionally, the specific auxiliary output $y^{*}$, which leads to the ISE-optimal response for set point changes, will be obtained.

Consider a controllable SISO $n$ th-order linear system of relative order $r$ with state space description:

$$
\begin{aligned}
& \dot{x}=A x+b u \\
& y=c x
\end{aligned}
$$

and transfer function description:

$$
\frac{y(s)}{u(s)}=\frac{\gamma_{1}+\gamma_{2} s+\ldots+\gamma_{n-r+1} s^{n-r}}{\alpha_{1}+\alpha_{2} s+\ldots+\alpha_{n} s^{n-1}+s^{n}}=\frac{c \operatorname{Adj}(s I-A) b}{\operatorname{det}(s I-A)}
$$

and assume that $A$ is Hurwitz and $c \operatorname{Adj}(s I-A) b$ has at least one RHP (right-half-plane) zero. Now consider an auxiliary output for the system (Eq. 2):

$$
y^{*}=c^{*} x
$$

which leads to the transfer function description:

$$
\frac{y^{*}(s)}{u(s)}=\frac{\gamma_{1}^{*}+\gamma_{2}^{*} s+\ldots+\gamma_{n-r_{+1}^{*}}^{*} s^{n-r^{*}}}{\alpha_{1}+\alpha_{2} s+\ldots+\alpha_{n} s^{n-1}+s^{n}}=\frac{c^{*} \operatorname{Adj}(s I-A) b}{\operatorname{det}(s I-A)}
$$

If Eq. 5 has all zeros in the LHP (left-half plane) and

$$
\gamma_{1}^{*}=\gamma_{1}
$$

then defining

$$
\begin{gathered}
G_{p}(s)=\frac{c \operatorname{Adj}(s I-A) b}{\operatorname{det}(s I-A)} \\
G_{p-}(s)=\frac{c^{*} \operatorname{Adj}(s I-A) b}{\operatorname{det}(s I-A)}
\end{gathered}
$$




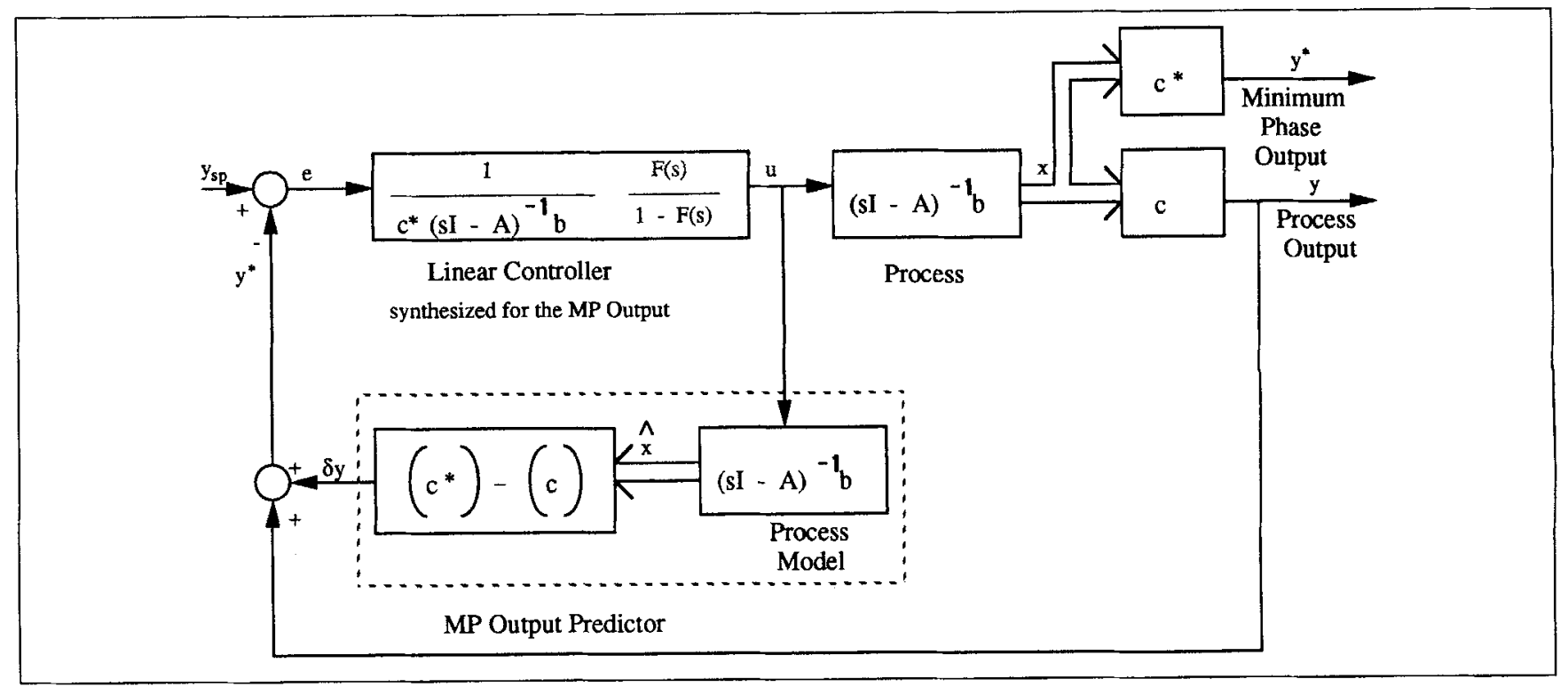

Figure 2. State space control structure for control of linear nonminimum-phase systems.

$$
G_{p+}(s)=\frac{c \operatorname{Adj}(s I-A) b}{c^{*} \operatorname{Adj}(s I-A) b}
$$

exactly the same decomposition is obtained as in the previous section. The $u-y^{*}$ system is stable and minimum phase, and the static gain between $y$ and $y^{*}$ is 1 . From Eq. 4, it becomes clear that $y^{*}$, which appears as an intermediate variable in Figure $1 b$, is actually an output to the process.

The parameterization of the controller may now be based on the minimum-phase $u-y^{*}$ system. For linear systems, a plethora of methods are available in the literature. One general method is the synthesis formula (see, for example, Smith and Corripio, 1985). If $F(s)$ is the desired closed transfer function between $y_{s p}$ and $y^{*}$, then the controller is given by:

$$
G_{c}(s)=\frac{\operatorname{det}(s I-A)}{c^{*} \operatorname{Adj}(s I-A) b} \cdot \frac{F(s)}{1-F(s)}
$$

To complete the structure, a corrective signal must be added in the feedback path to the measured nonminimum-phase output resulting in the minimum-phase output. This is necessary since the error given to the controller must be the difference between the minimum-phase output and the set point. The necessary corrective signal, $\delta y$, consists of the difference between the minimum-phase output map and the nonminimumphase output map based on values for the states obtained from a state observer:

$$
\delta y=c^{*} \hat{x}-c \hat{x}
$$

Since the process is open-loop stable, the states can be estimated on-line by an open-loop observer. The presence of an open-loop observer does not alter the closed-loop behavior of the system, under the assumption, of course, of a perfect model. The resulting controller structure is shown in Figure 2.

Compare Figure 2 with Figure 1a. It is exactly the same structure:
- The prediction of $y^{*}$ is exactly the same.

- The controller is synthesized for the $u-y^{*}$ system.

The structure in Figure 2 is more explicit in showing the various pieces and is expressed in terms of the state-space parameters, $A, b, c$, and $c^{*}$. Furthermore, as we will see in the next section, this structure allows a direct generalization to nonlinear systems.

The previously mentioned conditions on $y^{*}$ do not, however, result in a unique $y^{*}$. It is desirable to find the specific $y^{*}$ which results in the ISE optimal response for $y$ for set point changes. Through an appropriate change of coordinates, a companion form realization of Eq. 3 may be obtained:

$$
\begin{aligned}
\dot{\zeta}_{1} & =\zeta_{2} \\
\dot{\zeta}_{2} & =\zeta_{3} \\
& \vdots \\
\dot{\zeta}_{n-1} & =\zeta_{n} \\
\dot{\zeta}_{n} & =-\alpha_{1} \zeta_{1}-\alpha_{2} \zeta_{2}-\ldots-\alpha_{n} \zeta_{n}+u \\
y & =\gamma_{1} \zeta_{1}+\gamma_{2} \zeta_{2}+\ldots+\gamma_{n-r+1} \zeta_{n-r+1}
\end{aligned}
$$

This realization is convenient for two reasons:

- All information about the process zeros (number and location) is encoded in the output map.

- Problems of optimal regulation (in terms of an output error criterion) associated with Eq. 12 are of reduced dimensionality, $n-r$, and can be formulated as calculus of variations problems.

By definition, the integral square error (ISE) criterion is given by:

$$
\mathrm{ISE}=\frac{1}{2} \int_{0}^{\infty}[v-y(t)]^{2} d t
$$

where $v$ is a constant reference input. For the linear system of Eq. 12, the ISE criterion may be written equivalently as: 


$$
\mathrm{ISE}=\frac{1}{2} \int_{0}^{\infty}\left[v-\gamma_{1} \zeta_{1}-\gamma_{2} \zeta_{2}-\ldots-\gamma_{n-r+1} \zeta_{n-r+1}\right]^{2} d t
$$

The problem of minimizing the ISE subject to the dynamics (Eq. 12) has been solved for linear systems by Wonham and Johnson (1964); they showed that $\zeta_{1}(t), \zeta_{2}(t), \ldots$ are ISE optimal-state trajectories if and only if they satisfy:

$$
\sigma_{1} \zeta_{1}+\sigma_{2} \zeta_{2}+\ldots+\sigma_{n-r+1} \zeta_{n-r+1}=v
$$

where the coefficients $\sigma_{1}, \ldots, \sigma_{n-r+1}$ are such that the roots of the polynomial

$$
\sigma_{1}+\sigma_{2} s+\ldots+\sigma_{n-r+1} s^{n-r}
$$

are exactly the $(n-r)$ left-half-plane roots of the $2(n-r)$ degree polynomial

$$
\begin{aligned}
{\left[\gamma_{1}+\gamma_{2}(-s)+\ldots+\gamma_{n-r+1}\right.} & \left.(-s)^{n-r}\right] \\
& \times\left(\gamma_{1}+\gamma_{2} s+\ldots+\gamma_{n-r+1} s^{n-r}\right)
\end{aligned}
$$

and

$$
\sigma_{1}=\gamma_{1}
$$

Consider now the auxiliary output:

$$
y^{*}=\sigma_{1} \zeta_{1}+\sigma_{2} \zeta_{2}+\ldots+\sigma_{n-r+1} \zeta_{n-r+1} \text {. }
$$

Clearly, the zeros of the $u-y^{*}$ system are the roots of the polynomial of Eq. 16. By the above conditions, these are located at the left-half-plane zeros of the original system and the mirror images of the right-half-plane zeros with respect to the imaginary axis. Furthermore, since $\sigma_{1}=\gamma_{1}, y=y^{*}$ at steady state. The solution of the ISE optimization problem in the form of Eq. 15 implies that the ISE-optimal control of $y$ to $v$ is equivalent to the perfect control of $y^{*}$ to $v$. Back-transforming the expression of $\mathrm{Eq} .19$ to the original coordinates gives $y^{*}$ as a function of $x$ :

$$
y^{*}=c^{*} x
$$

The transfer function between $y$ and $y^{*}$ is given by:

$$
\frac{y(s)}{y^{*}(s)}=\frac{c \operatorname{Adj}(s I-A) b}{c^{*} \operatorname{Adj}(s I-A) b}=\frac{\gamma_{1}+\gamma_{2} s+\ldots+\gamma_{n-r+1} s^{n-r}}{\sigma_{1}+\sigma_{2} s+\ldots+\sigma_{n-r+1} s^{n-r}}
$$

which, because of the choice of $\sigma_{\imath}$ 's which leads to a cancellation of the left-half-plane zeros, reduces to an all-pass of order equal to the number of right-half-plane zeros in the original process. Clearly, the transfer function in Eq. 21 is exactly the ISE-optimal choice of $G_{p+}(s)$ given in the previous section. Thus, this $y^{*}$ is an auxiliary output whose perfect control will result in ISE-optimal control of $y$.

\section{Example 1}

To clarify the approach of this section, a second-order sys- tem with a RHP zero will be used as an example. Consider the following augmented system:

$$
\begin{aligned}
\dot{x}_{1} & =x_{2} \\
\dot{x}_{2} & =-\alpha_{1} x_{1}-\alpha_{2} x_{2}+u \\
y & =c_{1} x_{1}+c_{2} x_{2} \\
y^{*} & =c_{1} x_{1}-c_{2} x_{2}
\end{aligned}
$$

where $\alpha_{1}>0, \alpha_{2}>0, c_{1} / c_{2}<0$ and $y^{*}$ has been constructed by the method detailed in this section. The transfer function for the $\boldsymbol{u}-\boldsymbol{y}$ system is given by:

$$
\frac{y(s)}{u(s)}=\frac{c_{1}+c_{2} s}{\alpha_{1}+\alpha_{2} s+s^{2}}
$$

This system has a zero at $z=-c_{1} / c_{2}$, which is in the RHP. The transfer function for the $u-y^{*}$ system is:

$$
\frac{y^{*}(s)}{u(s)}=\frac{c_{1}-c_{2} s}{\alpha_{1}+\alpha_{2} s+s^{2}}
$$

which has a zero at $-z$, which is in the LHP since $z>0$, and is the mirror image of the zero in the $u-y$ system. The transfer function between $y$ and $y^{*}$ is given by:

$$
\frac{y(s)}{y^{*}(s)}=\frac{c_{1}+c_{2} s}{c_{1}-c_{2} s}
$$

which is an all-pass. The corrective signal that must be added to the output measurement in the feedback path to obtain the minimum-phase output is:

$$
\delta y=c^{*} x-c x=-2 c_{2} x_{2}
$$

Choosing the desired closed-loop transfer function, $F(s)$, between $y^{*}(s)$ and $y_{s p}(s)$ as:

$$
F(s)=\frac{1}{\epsilon S+1}
$$

where $\epsilon$ is a tunable parameter, results in the following transfer function from $y_{s p}$ to $y$ :

$$
\frac{y(s)}{y_{s p}(s)}=\frac{c_{1}+c_{2} s}{\left(c_{1}-c_{2} s\right)(\epsilon s+1)}
$$

which is a first-order lag plus an all-pass.

In conclusion, the steps outlined in this section are:

- Define an auxiliary output, $y^{*}$, which has the same static gain as $y$, and the $u-y^{*}$ system is stable and minimum phase. In particular, choose the auxiliary output $y^{*}$ such that the solution of the ISE-optimal control problem can be expressed as the perfect control in $y^{*}$.

- Parameterize a controller based on the minimum-phase $u-y^{*}$ system.

- Add the necessary corrective signal in the feedback path to obtain an on-line estimate of $y^{*}$. 
These steps will be addressed in the next sections for nonlinear systems.

\section{Statically Equivalent Outputs}

The auxiliary output, $y^{*}$, of the previous section made the $u-y^{*}$ system minimum phase and had the same static gain as the system with the original output, $y$. In a linear setting, two outputs $y$ and $y^{*}$ can be called statically equivalent, if they have the same static gain. The system with $y$ as the output will automatically have the same poles as the system with $y^{*}$ as the output, but the zeros will be different. This notion will now be introduced for the first time in a general nonlinear setting. In the next section, the minimum-phase output predictor structure for nonlinear systems will be developed based on this notion. Rigorous mathematical formulations of the following concept of static equivalence are available in Wright (1990).

\section{Definition 1}

Consider the SISO nonlinear system:

$$
\begin{aligned}
\dot{x} & =f(x)+g(x) u \\
y & =h(x) \\
y^{*} & =h^{*}(x)
\end{aligned}
$$

and denote by $E$ its equilibrium set:

$$
E=\left\{x \in \boldsymbol{R}^{n} \mid \text { there is } \lambda \in R \text { such that } f(x)+\lambda g(x)=0\right\}
$$

The outputs $y$ and $y^{*}$ are called statically equivalent if

$$
h(x)=h^{*}(x) \text { for every } x \in E
$$

Since the equilibrium set $E$ is unaffected by the choice of output, an immediate consequence of the definition is that the input/output relationships at equilibrium of the two staticallyequivalent outputs are the same. Consequently, the static gains of the two statically-equivalent outputs are the same. From the previous discussion, it is clear that the $y^{*}$ defined in the linear case is a statically-equivalent output. This concept will be useful in developing a control structure for nonlinear, nonminimum-phase systems.

\section{Minimum-Phase Output Predictor for Nonlinear Systems}

A general control structure for nonminimum-phase compensation of nonlinear systems will now be developed containing the components shown in Figure 3, which is completely analogous to the linear control structure of Figure 2. The structure is based on an a priori choice of a statically-equivalent, minimum-phase output, $y^{*}$, which is estimated on-line and controlled to set point. Because of static equivalence, $y$ and $y^{*}$ have the same set point and, therefore, $y$ is also controlled to set point. The performance characteristics of the closed-loop response of $y$ will, of course, depend on the choice of $y^{*}$. Whenever ISE optimization results are available (in terms of the output $y$ ), it is possible to appropriately choose

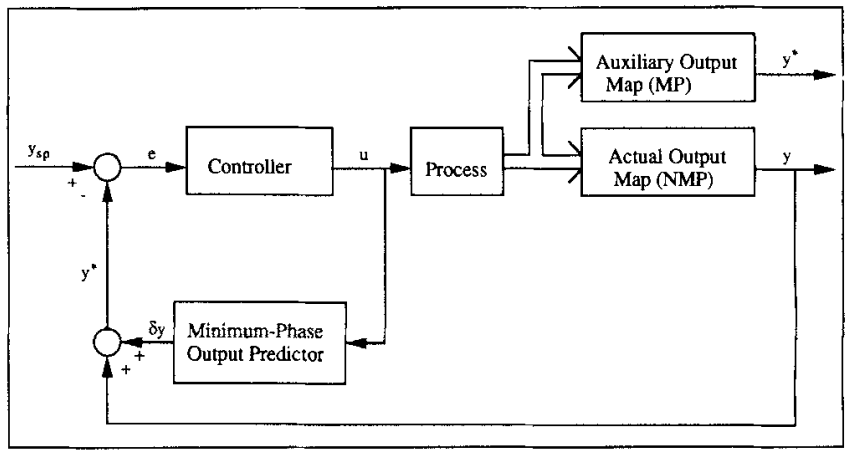

Figure 3. General structure of minimum-phase output predictor.

$y^{*}$ so that the closed-loop response in $y$ asymptotically approaches the ISE optimal response.

Consider a general open-loop stable SISO nonlinear system:

$$
\begin{gathered}
\dot{x}=f(x)+g(x) u \\
y=h(x)
\end{gathered}
$$

with relative order $r$. It is assumed that the system (Eq. 29) is nonminimum phase (that is, it has unstable zero dynamics in the sense of Byrnes and Isidori, 1985). Consider also an auxiliary output

$$
y^{*}=h^{*}(x)
$$

which, when combined with the state equations of Eq. 29: is statically equivalent to $y=h(x)$, induces asymptotically stable zero dynamics (the $u-y^{*}$ system is minimum-phase), and has relative order $r^{*}$. A controller for the minimum-phase $u-y^{*}$ system can be easily synthesized using state space methods. In particular, consider the input/output linearizing state feedback (Kravaris and Chung, 1987):

$$
u=\frac{v-h^{*}(x)-\beta_{1} L_{f} h^{*}(x)-\ldots-\beta_{r}^{*} L_{f}^{r^{*}} h^{*}(x)}{\beta_{r}^{*} L_{g} L_{f}^{r^{*}-1} h^{*}(x)}
$$

where $\beta_{1}, \ldots, \beta_{r}^{*}$ are scalar parameters. The closed-loop response will then satisfy:

$$
y^{*}+\beta_{1} \frac{d y^{*}}{d t}+\ldots+\beta_{r}^{*} \frac{d^{r^{*}} y^{*}}{d t^{r^{*}}}=v
$$

This controller will provide closed-loop internal asymptotic stability and asymptotic tracking of $y^{*}=v$, as long as the roots of the polynomial

$$
B(\xi)=1+\beta_{1} \xi+\ldots+\beta_{r^{*}} \xi^{r^{*}}
$$

are in the left-half plane (Kravaris, 1988). This static feedback, when combined with an open-loop observer

$$
\dot{\hat{x}}=f(\hat{x})+g(\hat{x}) u
$$

and an external linear controller, yields an output feedback 


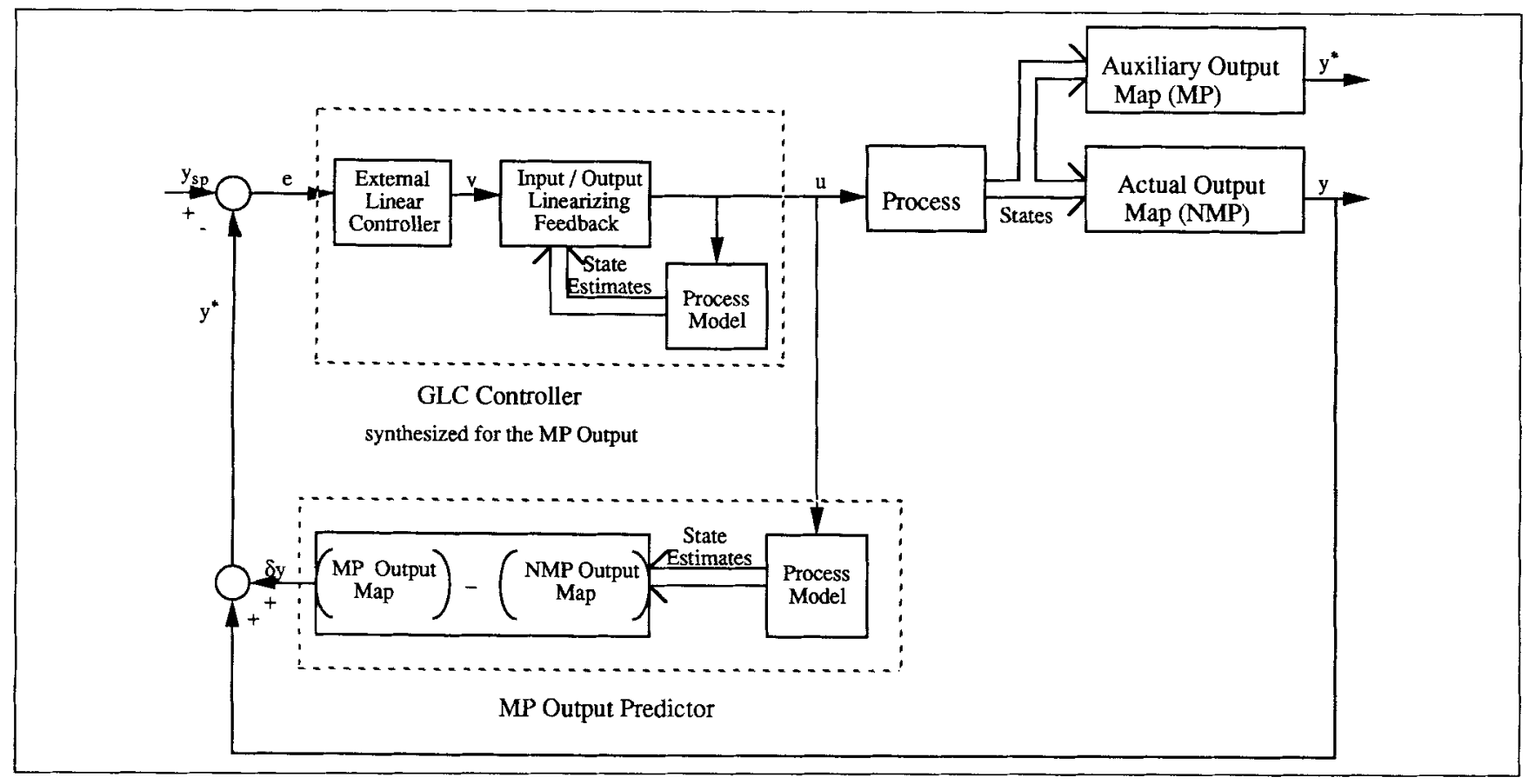

Figure 4. Complete minimum-phase output predictor structure for nonlinear systems.

controller for the $u-y^{*}$ system (GLC controller). To complete the control structure, it is necessary to add a corrective signal to the measured nonminimum-phase output, $y$, of the process, resulting in an on-line estimate of $y^{*}$. This corrective signal is given by:

$$
\delta y=h^{*}(\hat{x})-h(\hat{x})
$$

where the state values are obtained from the open-loop observer (Eq. 34). The complete control structure is shown in Figure 4.

Note that the control structure in Figure 4 is a direct generalization of the one in Figure 2 to nonlinear systems. Also, it must be emphasized that the choice of the auxiliary output, $y^{*}=h^{*}(x)$, is completely arbitrary, as long as it is minimumphase and statically-equivalent. However, the performance characteristics of the control system will depend strongly on the choice of $y^{*}$, and this motivates searching for the particular choice of $y^{*}$ which provides optimal response in $y$ when perfectly controlled to set point. In the next section, this problem will be addressed in the context of the ISE performance criterion; the appropriate necessary condition for ISE optimality will be derived as well as closed-form expressions for the ISEoptimal output in special cases.

The proposed control structure provides a closed-loop input/ output behavior which is linear in $y^{*}$, but generally nonlinear in $y$. This is in agreement with the intuitive expectation that the closed-loop response must inherit the right-half-plane zeros of the process, and since these will be generally nonlinear, the closed-loop response will have to be nonlinear as well. There are, of course, special cases of nonlinear systems with linear right-half-plane zeros (Nikolaou and Manousiouthakis, 1990); for these it is possible to obtain a linear closed-loop response in $y$ by appropriate choice of $h^{*}$.

\section{Formulation of the ISE Optimization Problem for Nonlinear Systems in Natural Coordinates: Prop- erties of the Optimal Response}

The generalization of linear ISE optimization results to nonlinear systems is not a trivial task. A general solution is not yet available in the literature for systems of order higher than 2. However, for the class of nonlinear systems which may be described in natural coordinates, it is possible to obtain specific results. The properties of the natural coordinate description of nonlinear systems, which is the nonlinear analog of the realization of Eq. 12, are reviewed in Appendix A. In this section, general ISE optimization results will be derived for nonlinear systems in natural coordinates. These will generalize the linear state-space results given in a previous section.

Consider a nonlinear system of relative order, $r$, in natural coordinates. This is of the form (see Appendix A):

$$
\begin{aligned}
\dot{\zeta}_{1} & =\zeta_{2} \\
\dot{\zeta}_{2} & =\zeta_{3} \\
& \vdots \\
\dot{\zeta}_{n-1} & =\zeta_{n} \\
\dot{\zeta}_{n} & =\varphi(\zeta)+\psi(\zeta) u \\
y & =h\left(\zeta_{1}, \ldots, \zeta_{n-r+1}\right)
\end{aligned}
$$

where

$$
\frac{\partial h}{\partial \zeta_{n-r+1}}(\zeta) \neq 0
$$

As shown in Appendix A (Proposition A1), the system of Eq. 
36 is locally minimum phase if and only if the roots of the polynomial in $\xi$,

$\frac{\partial h}{\partial \zeta_{1}}(\zeta)+\frac{\partial h}{\partial \zeta_{2}}(\zeta) \xi+\ldots+\frac{\partial h}{\partial \zeta_{n-r}}(\zeta) \xi^{n-r-1}+\frac{\partial h}{\partial \zeta_{n-r+1}}(\zeta) \xi^{n-r}$

are in the open left half plane for every $\zeta$ in a neighborhood of the equilibrium point.

The problem of minimizing the ISE criterion (Eq. 13) subject to the dynamics of Eq. 36 is a singular optimal control problem, because its Hamiltonian is linear in $u$. Moreover, in the absence of constraints on $u$, it is not a well-posed problem [the optimal $u(t)$ must have impulsive behavior at $t=0$ ]. Although techniques are available to approach singular optimal control problems (Johnson and Gibson, 1963; Johnson, 1965), it is much more convenient to transform it to a nonsingular problem following the method of Kelley (1964). The natural coordinate system provides exactly the appropriate dynamic description for this purpose.

Since the output is a function of $\zeta_{1}, \ldots, \zeta_{n-r+1}$ only and, therefore, only the first $n-r$ state equations are relevant in calculating the optimal solution, the remaining states represent ignorable coordinates. Consequently, the dimensionality of the ISE-optimal control problem can be reduced and it can be formulated as follows:

Minimize

$$
\text { ISE }=\frac{1}{2} \int_{0}^{\infty}\left[v-h\left(\zeta_{1}, \ldots, \zeta_{n-r+1}\right)\right]^{2} d t
$$

subject to the dynamics

$$
\begin{array}{ccc}
\dot{\zeta}_{1} & =\zeta_{2} & \zeta_{1}(0)=\zeta_{1, o} \\
\vdots & \vdots \\
\zeta_{n-r}=\zeta_{n-r+1} & \zeta_{n-r}(0)=\zeta_{n-r, o}
\end{array}
$$

and the terminal conditions

$$
\begin{gathered}
h\left(\zeta_{1}(\infty), 0, \ldots, 0\right)=v \\
\zeta_{2}(\infty)=0 \\
\vdots \\
\zeta_{n-r}(\infty)=0
\end{gathered}
$$

This is a nonsingular optimal control problem with $\zeta_{n-r+1}$ as the input and $\zeta_{1}, \ldots, \zeta_{n-r}$ as states. Its solution will provide the optimal path that takes the system from a given initial point (with coordinates $\zeta_{1, a}, \ldots, \zeta_{n-r, o}$ ) to a given final equilibrium point (the one that corresponds to $y=v$ ).

Motivated from the discussion on linear systems, it is not the ISE-optimal trajectories that are sought. What is desired to calculate is an auxiliary output,

$$
y^{*}=h^{*}\left(\zeta_{1}, \zeta_{2}, \ldots, \zeta_{n-r}, \zeta_{n-r+1}\right)
$$

whose perfect control to $v$ induces the ISE-optimal state trajectories. This leads to the following definition.

\section{Definition 2}

Consider a system of the form of Eq. 36 and let

$$
y^{*}=h^{*}\left(\zeta_{1}, \zeta_{2}, \ldots, \zeta_{n-r}, \zeta_{n-r+1}\right)
$$

be an auxiliary output with

$$
\frac{\partial h^{*}}{\partial \zeta_{n-r+1}}(\zeta) \neq 0
$$

Furthermore, let

$$
\zeta_{n-r+1}=\Omega^{*}\left(\zeta_{1}, \ldots, \zeta_{n-r}, y^{*}\right)
$$

be the implicit function defined locally as the solution of $\mathrm{Eq}$. 38 for $\zeta_{n-r+1}$.

The output $y^{*}=h^{*}(\zeta)$ will be said to be ISE-optimal, if for every $\zeta_{1, o}, \ldots, \zeta_{n-r, o}$ and every $v$, the trajectories obtained as the solution of

$$
\begin{array}{ccrl}
\dot{\zeta}_{1} & =\zeta_{2} & \zeta_{1}(0) & =\zeta_{1, o} \\
\vdots & \vdots \\
\dot{\zeta}_{n-r} & =\Omega^{*}\left(\zeta_{1}, \ldots, \zeta_{n-r}, v\right) & \zeta_{n-r}(0) & =\zeta_{n-r, o}
\end{array}
$$

are exactly the ISE-optimal trajectories.

\section{Remark 1}

A similar notion of ISE-optimal outputs could be defined for general nonlinear systems which are not in natural coordinates. The natural coordinate description and the resulting order reduction of the ISE-optimization problem makes the formulation of the notion much more explicit.

\section{Theorem 1}

Consider a dynamic system of the form of Eq. 36. If the auxiliary output $y^{*}=h^{*}\left(\zeta_{1}, \zeta_{2}, \ldots, \zeta_{n-r}, \zeta_{n-r+1}\right)$ is ISE-optimal, then it is statically equivalent to $y=h\left(\zeta_{1}, \zeta_{2}, \ldots, \zeta_{n-r}, \zeta_{n-r+1}\right)$ and it induces asymptotically stable zero dynamics, that is, the dynamic system

$$
\begin{aligned}
\dot{\zeta}_{1} & =\zeta_{2} \\
\dot{\zeta}_{2} & =\zeta_{3} \\
& \vdots \\
\dot{\zeta}_{n-1} & =\zeta_{n} \\
\dot{\zeta}_{n} & =\varphi(\zeta)+\psi(\zeta) u \\
y^{*} & =h^{*}\left(\zeta_{1}, \zeta_{2}, \ldots, \zeta_{n-r}, \zeta_{n-r+1}\right)
\end{aligned}
$$

is minimum phase.

The proof of Theorem 1 is in Appendix B. Theorem 1 establishes the intuitively expected result that an ISE-optimal output will automatically be statically equivalent and minimum phase. Thus, it can be used in the minimum-phase output predictor structure of the previous section leading to ISE- 
optimal, closed-loop dynamics in the limit of perfect control of $y^{*}$ to set point.

The key question is, of course, how to find an ISE-optimal output. It is known from optimal control theory that Pontryagin's theorem offers a set of necessary conditions that the optimal control trajectories must satisfy. These conditions will now be recast in terms of an output function in the following theorem.

\section{Theorem 2: necessary condition for ISE optimality}

Consider a dynamic system of the form of Eq. 36. If the output $y^{*}=h^{*}\left(\zeta_{1}, \zeta_{2}, \ldots, \zeta_{n-r+1}\right)$ is ISE-optimal, then the expression,

$$
h^{*}\left(\zeta_{1}, \frac{d \zeta_{1}}{d t}, \ldots, \frac{d^{n-r} \zeta_{1}}{d t^{n-r}}\right)=v
$$

is an $(n-r)$ th integral of the $2(n-r)$ order Euler-Lagrange equation

$$
\begin{aligned}
\sum_{k=1}^{n-r+1}(-1)^{k-1} \frac{d^{k-1}}{d t^{k-1}} & \left\{\frac{\partial h}{\partial \zeta_{k}}\left(\zeta_{1}, \frac{d \zeta_{1}}{d t}, \ldots, \frac{d^{n-r} \zeta_{1}}{d t^{n-r}}\right)\right. \\
\times & {\left.\left[v-h\left(\zeta_{1}, \frac{d \zeta_{1}}{d t}, \ldots, \frac{d^{n-r} \zeta_{1}}{d t^{n-r}}\right)\right]\right\}=0, }
\end{aligned}
$$

that meets the $(n-r)$ terminal time conditions:

$$
\begin{gathered}
h\left(\zeta_{1}(\infty), 0, \ldots, 0\right)=v \\
\zeta_{2}(\infty)=0 \\
\vdots \\
\zeta_{n-r}(\infty)=0
\end{gathered}
$$

The proof of Theorem 2 is in Appendix B. The result of Theorem 2 establishes that the calculation of an ISE-optimal output (assuming that it exists) reduces to the calculation of an $(n-r)$ th integral of the Euler-Lagrange equation (Eq. 40), whose $(n-r)$ arbitrary constants are fixed via the terminal conditions (Eq. 41). Analytical calculation of this integral is possible only in special cases, leading to analytical expressions for the ISE-optimal output; these are given in the corollary that follows.

\section{Corollary}

a) If the system of Eq. 36 is minimum phase, then the ISEoptimal output is given by:

$$
h^{*}(\zeta)=h(\zeta)
$$

b) If the system of Eq. 36 has relative order $r=n-1$ and it is nonminimum phase,

$$
\frac{\frac{\partial h}{\partial \zeta_{1}}(\zeta)}{\frac{\partial h}{\partial \zeta_{2}}(\zeta)}>0
$$

then the ISE-optimal output is given by:

$$
h^{*}\left(\zeta_{1}, \zeta_{2}\right)=h\left(\zeta_{1}, \zeta_{2}\right)-2 \zeta_{2} \frac{\partial h}{\partial \zeta_{2}}\left(\zeta_{1}, \zeta_{2}\right)
$$

The proof of the corollary is in Appendix B.

\section{Remark 2}

The case $n=2, r=1$, which is a special case of the one in part $b$ of the Corollary, has been studied by Kravaris and Daoutidis (1990) in the framework of singular optimal control theory. It was shown that the ISE-optimal trajectories must lie on the singular arc:

$$
h\left(\zeta_{1}, \zeta_{2}\right)-2 \zeta_{2} \frac{\partial h}{\partial \zeta_{2}}\left(\zeta_{1}, \zeta_{2}\right)=v
$$

This is, of course, consistent with the result of the Corollary and at the same time indicates an intrinsic connection between the notion of ISE-optimal output and the notion of singular surface in singular optimal control theory.

\section{Remark 3}

The results of Theorems 1 and 2 are entirely consistent with the results for linear systems presented earlier. For a linear system, the ISE-optimal output (Eq. 38) can be calculated analytically and is given by Eq. 19. The characteristic equation of the Euler-Lagrange equation (Eq. 40) is the polynomial of degree $2 n-2 r$ given in Eq. 17. The ISE-optimal output must be minimum phase in the sense that the roots of Eq. 16 lie in the left-half plane, must satisfy the static equivalence property (Eq. 18) and at the same time generate a $(n-r)$ th integral of the Euler-Lagrange equation (Eq. 40).

In the general (nonlinear) case, the appropriate $(n-r)$ th integral of the Euler-Lagrange equation must be determined numerically. Finally, it should be pointed out that for the general case where analytical solutions are not available, there are mathematical questions of existence and uniqueness of the sought for integral of the Euler-Lagrange equation, for which answers are not available at the moment.

\section{Example 2}

To clarify the entire approach for nonlinear systems, a nonlinear, second-order, nonminimum-phase system will be used as an example. Consider first the following augmented system:

$$
\begin{aligned}
\dot{x}_{1} & =x_{2} \\
\dot{x}_{2} & =f\left(x_{1}, x_{2}\right)+g\left(x_{1}, x_{2}\right) u \\
y & =h\left(x_{1}, x_{2}\right) \\
y^{*} & =h^{*}\left(x_{1}, x_{2}\right)
\end{aligned}
$$

where it is assumed that 


$$
\begin{gathered}
g\left(x_{1}, x_{2}\right) \neq 0 \\
\frac{\partial h}{\partial x_{2}}\left(x_{1}, x_{2}\right) \neq 0 .
\end{gathered}
$$

The relative order of this system is then $r=n-1=1$, and it will be nonminimum phase if, and only if,

$$
\frac{\frac{\partial h}{\partial x_{1}}\left(x_{1}, x_{2}\right)}{\frac{\partial h}{\partial x_{2}}\left(x_{1}, x_{2}\right)}<0
$$

From the solution for systems of relative order $r=n-1, \mathrm{Eq}$. 43 , the ISE-optimal output for the system may be determined:

$$
y^{*}=h^{*}\left(\hat{x}_{1}, \hat{x}_{2}\right)=h\left(\hat{x}_{1}, \hat{x}_{2}\right)-2 \hat{x}_{2} \frac{\partial h}{\partial x_{2}}\left(\hat{x}_{1}, \hat{x}_{2}\right) .
$$

The GLC state feedback (Eq. 31) is then given by

$$
u=\frac{v-h^{*}\left(x_{1}, x_{2}\right)-\beta_{1}\left(x_{2} \frac{\partial h^{*}}{\partial x_{1}}+f\left(x_{1}, x_{2}\right) \frac{\partial h^{*}}{\partial x_{2}}\right)}{\beta_{1} g\left(x_{1}, x_{2}\right) \frac{\partial h^{*}}{\partial x_{2}}}
$$

and the corrective signal which must be added to the nonminimum-phase output to get the minimum-phase output is:

$$
\delta y=h^{*}(x)-h(x)=-2 x_{2} \frac{\partial h}{\partial x_{2}}\left(x_{1}, x_{2}\right)
$$

Consider now the specific output map

$$
h\left(x_{1}, x_{2}\right)=x_{1}-3 x_{2}-x_{2}^{3}
$$

which leads to a nonminimum-phase system because

$$
\frac{\frac{\partial h}{\partial x_{1}}\left(x_{1}, x_{2}\right)}{\frac{\partial h}{\partial x_{2}}\left(x_{1}, x_{2}\right)}=\frac{-1}{3\left(1+x_{2}^{2}\right)}<0
$$

The ISE-optimal, statically-equivalent, minimum-phase output of the system is found from Eq. 46:

$$
y^{*}=h^{*}\left(x_{1}, x_{2}\right)=x_{1}+3 x_{2}+5 x_{2}^{3} .
$$

The GLC state feedback is then calculated from Eq. 47:

$$
u=\frac{v-\left(x_{1}+3 x_{2}+5 x_{2}^{3}\right)-\beta_{1}\left(x_{2}+f\left(x_{1}, x_{2}\right)\left(3+15 x_{2}^{2}\right)\right)}{\beta_{1} g\left(x_{1}, x_{2}\right)\left(3+15 x_{2}^{2}\right)}
$$

The resulting closed-loop system is then given by:

$$
\begin{aligned}
\dot{x}_{1} & =x_{2} \\
\dot{x}_{2} & =\frac{v-x_{1}-3 x_{2}-5 x_{2}^{3}-\beta_{1} x_{2}}{\beta_{1}\left(3+15 x_{2}^{2}\right)} \\
y & =x_{1}-3 x_{2}-x_{2}^{3} \\
y^{*} & =x_{1}+3 x_{2}+5 x_{2}^{3} .
\end{aligned}
$$

From this it is easy to verify that:

$$
y^{*}+\beta_{1} \frac{d y^{*}}{d t}=v
$$

or

$$
\frac{y^{*}(s)}{v(s)}=\frac{1}{1+\beta_{1} s}
$$

which implies that the response will be ISE-optimal in the limit as $\beta_{1} \rightarrow 0$.

\section{Computer Simulations}

The utility of the minimum-phase output predictor structure will now be illustrated using a chemical engineering example. Consider a stirred tank reactor, where the isothermal series/ parallel Van de Vusse reaction (Van de Vusse, 1964; Kantor, 1986; Kravaris and Daoutidis, 1990) is taking place:

$$
\begin{aligned}
& A-B-C \\
& 2 A \rightarrow D
\end{aligned}
$$

The rates of formation of $A$ and $B$ are:

$$
\begin{aligned}
& r_{A}=-k_{1} c_{A}-k_{3} c_{A}^{2} \\
& r_{B}=k_{1} c_{A}-k_{2} c_{B}
\end{aligned}
$$

where $k_{1}=50 \mathrm{~h}^{-1}, k_{2}=100 \mathrm{~h}^{-1}$, and $k_{3}=10 \mathrm{~L} / \mathrm{mol} \cdot \mathrm{h}$ are the reaction rate constants. The feed stream consists of pure $A$. The mass balances for $A$ and $B$ are given by:

$$
V \frac{d c_{A}}{d t}=F\left(c_{A o}-c_{A}\right)+V\left(-k_{1} c_{A}-k_{3} c_{A}^{2}\right)
$$

$$
V \frac{d c_{B}}{d t}=F\left(-c_{B}\right)+V\left(k_{1} c_{A}-k_{2} c_{B}\right)
$$

where $F$ is the inlet flow rate of $A, V$ is the reactor volume, which is kept constant during the operation, and $c_{A}$ and $c_{B}$ are the concentrations of $A$ and $B$ inside the reactor, respectively. The concentration of $\boldsymbol{A}$ in the feed stream is given by $c_{A o}$ and is equal to $10 \mathrm{~mol} / \mathrm{L}$. It is desired to maintain $c_{B}$ at a constant value by manipulating the dilution rate, $F / V$. Initially, the system is at steady state with $c_{A s}=3.0 \mathrm{~mol} / \mathrm{L}$ and $c_{B s}=1.117$ $\mathrm{mol} / \mathrm{L}$. The system may be put into standard state space form by letting: 


$$
\begin{gathered}
x_{1}=c_{A} \\
x_{2}=c_{B} \\
u=\frac{F}{V} \\
y=c_{B}
\end{gathered}
$$

which results in

$$
\begin{aligned}
& \dot{x}_{1}=-k_{1} x_{1}-k_{3} x_{1}^{2}+\left(c_{A o}-x_{1}\right) u \\
& \dot{x}_{2}=k_{1} x_{1}-k_{2} x_{2}-x_{2} u \\
& y=x_{2}
\end{aligned}
$$

This particular system is not globally nonminimum phase. The sign of

$$
k_{2}-k_{1}-2 k_{3} x_{1}+k_{1} \frac{c_{A o}-2 x_{1}}{x_{2}}
$$

locally around the steady state will determine whether the system is minimum or nonminimum phase (Daoutidis and Kravaris, 1990). For the parameters and steady-state values used in this example, the system is in the nonminimum-phase region.

The coordinate transformation

$$
\begin{aligned}
& \zeta_{1}=\frac{c_{A o}-x_{1}}{x_{2}} \\
& \zeta_{2}=\frac{k_{1} x_{1}+k_{3} x_{1}^{2}}{x_{2}}+\frac{\left(x_{1}-c_{A o}\right)\left(k_{1} x_{1}-k_{2} x_{2}\right)}{x_{2}^{2}}
\end{aligned}
$$

puts the original system into natural coordinate form. Applying Eq. 46 to calculate $y^{*}$ and back-transforming into the original coordinates yields:

$y^{*}=h^{*}\left(x_{1}, x_{2}\right)=x_{2}-\frac{D\left(x_{1}, x_{2}\right)}{A\left(x_{1}, x_{2}\right)}$

$$
+\frac{B\left(x_{1}, x_{2}\right) D\left(x_{1}, x_{2}\right)}{A\left(x_{1}, x_{2}\right)\left(2 A\left(x_{1}, x_{2}\right) x_{2}+B\left(x_{1}, x_{2}\right)\right)}
$$

where

$A\left(x_{1}, x_{2}\right)=\frac{k_{3} c_{A o}^{2}-2 k_{3} c_{A o} x_{1}+k_{3} x_{1}^{2}}{x_{2}^{2}}$

$B\left(x_{1}, x_{2}\right)=$

$\frac{k_{1} c_{A o}^{2}-k_{1} c_{A o} x_{1}-k_{1} c_{A o} x_{2}-2 k_{3} c_{A o}^{2} x_{2}+2 k_{3} c_{A o} x_{1} x_{2}+k_{3} x_{1}^{2} x_{2}}{x_{2}^{2}}$

$D\left(x_{1}, x_{2}\right)=\frac{k_{1} x_{1}+k_{3} x_{1}^{2}}{x_{2}}+\frac{\left(x_{1}-c_{A o}\right)\left(k_{1} x_{1}-k_{2} x_{2}\right)}{x_{2}^{2}}$

The GLC state feedback can then be computed for the $u-y^{*}$ system using Eq. 47:

$$
u=\frac{v-h^{*}\left(x_{1}, x_{2}\right)-\beta_{1}\left[f_{1}\left(x_{1}, x_{2}\right) \frac{\partial h^{*}}{\partial x_{1}}+f_{2}\left(x_{1}, x_{2}\right) \frac{\partial h^{*}}{\partial x_{2}}\right]}{\beta_{1}\left[g_{1}\left(x_{1}, x_{2}\right) \frac{\partial h^{*}}{\partial x_{1}}+g_{2}\left(x_{1}, x_{2}\right) \frac{\partial h^{*}}{\partial x_{2}}\right]}
$$

where

$$
\begin{aligned}
& f_{1}\left(x_{1}, x_{2}\right)=-k_{1} x_{1}-k_{3} x_{1}^{2} \\
& f_{2}\left(x_{1}, x_{2}\right)=k_{1} x_{1}-k_{2} x_{2} \\
& g_{1}\left(x_{1}, x_{2}\right)=c_{A 0}-x_{1} \\
& g_{2}\left(x_{1}, x_{2}\right)=-x_{2}
\end{aligned}
$$

For this example, a PI controller is used for the linear external controller in the GLC structure for $y^{*}$. The corrective signal that must be added to the measured output to complete the minimum-phase output predictor structure is given by Eq. 48 , which for the present system becomes:

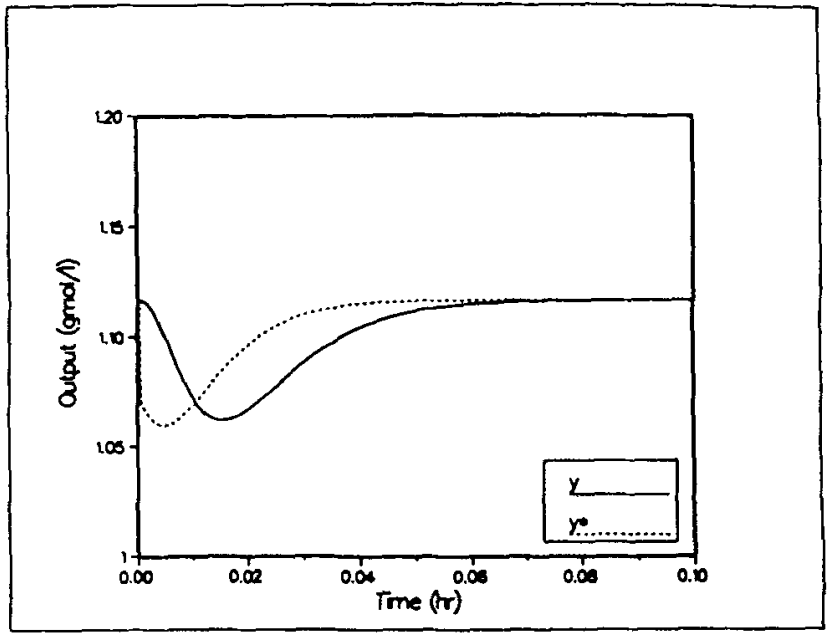

(a)

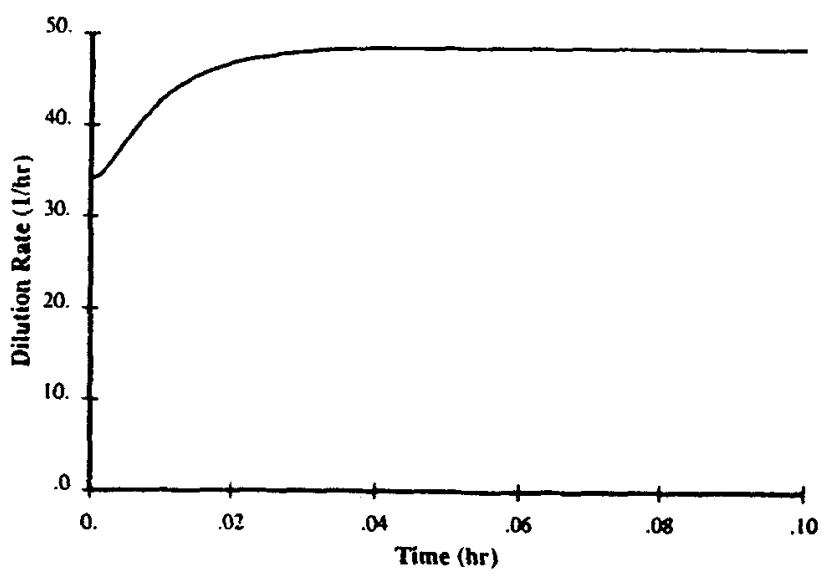

(b)

Figure 5. Closed-loop response (a) calculated values of manipulated input (b) in response to a step disturbance in the inlet concentration. 


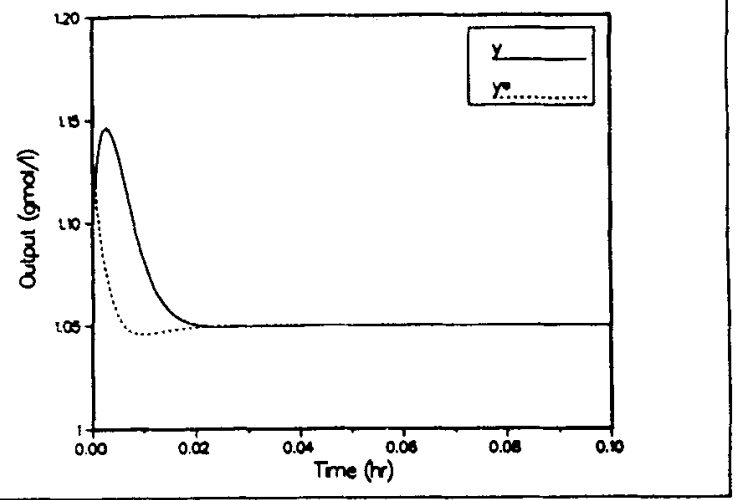

(a)

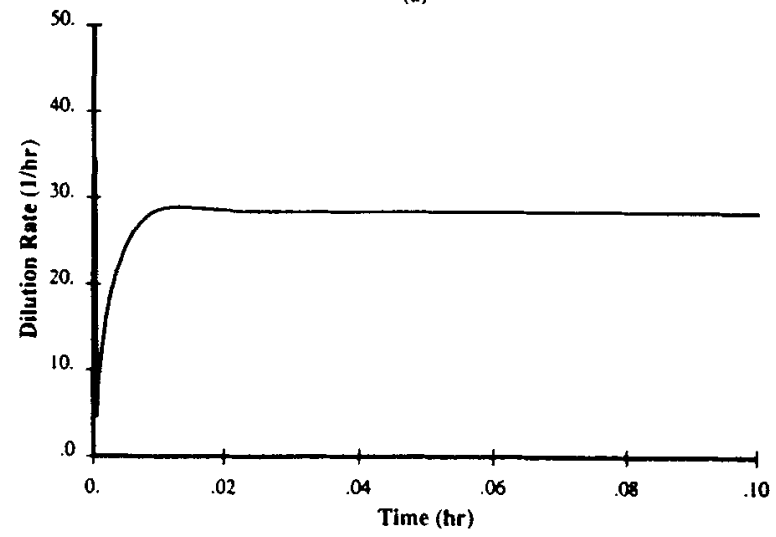

(b)

Figure 6. Closed-loop response (a) calculated values of manipulated input (b) in response to a step change in the set point value of the output.

$$
\delta y=-\frac{D\left(\hat{x}_{1}, \hat{x}_{2}\right)}{A\left(\hat{x}_{1}, \hat{x}_{2}\right)}+\frac{B\left(\hat{x}_{1}, \hat{x}_{2}\right) D\left(\hat{x}_{1}, \hat{x}_{2}\right)}{A\left(\hat{x}_{1}, \hat{x}_{2}\right)\left(2 A\left(\hat{x}_{1}, \hat{x}_{2}\right) \hat{x}_{2}+B\left(\hat{x}_{1}, \hat{x}_{2}\right)\right]}
$$

where $A, B$, and $D$ are the same as given above, but uses state estimates obtained from:

$$
\begin{aligned}
& \dot{\hat{x}_{1}}=-k_{1} \hat{x}_{1}-k_{3} \hat{x}_{1}^{2}+\left(c_{A o}-\hat{x}_{1}\right) u \\
& \dot{\hat{x}}_{2}=k_{1} \hat{x}_{1}-k_{2} \hat{x}_{2}-\hat{x}_{2} u .
\end{aligned}
$$

The response of the closed-loop system was simulated for three different situations. The gains of the external PI controller are given as $K_{c}$ and $\tau_{l}$. First, a step disturbance in $c_{A o}$ from its steady-state value of $10 \mathrm{~mol} / \mathrm{L}$ to $9 \mathrm{~mol} / \mathrm{L}$ was examined. The closed-loop response for both $y$ and $y^{*}$ is shown in Figure 5a, for $\beta_{1}=0.01, K_{c}=5.0$, and $\tau_{t}=10.0$. Note the difference in dynamics of $y$ and $y^{*}$. The required change in the manipulated variable is shown in Figure 5b. Figure 6a shows the closedloop response, and Figure $6 \mathrm{~b}$ shows the corresponding function of the manipulated variable for a negative step change in the set point value in the output, from 1.117 to 1.050 , and the same controller gains. The closed-loop response to errors in the initial conditions of the state estimators is shown in Figure $7 \mathrm{a}$, and the corresponding response of the manipulated variable

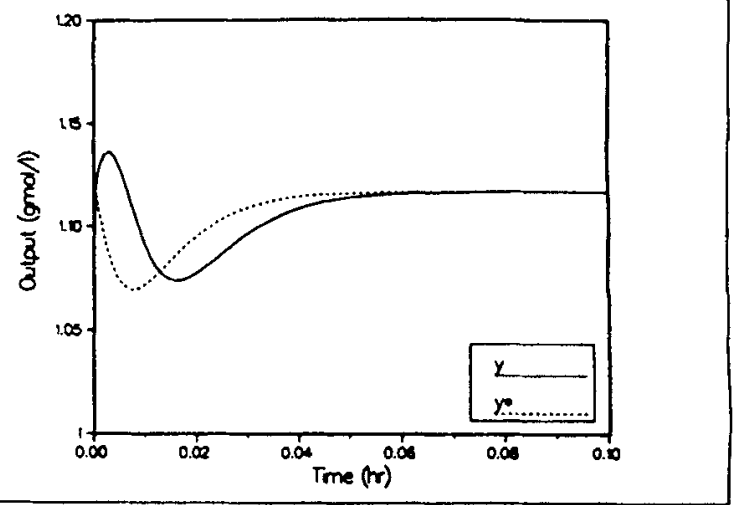

(ii)



Figure 7. Closed-loop response (a) calculated values of manipulated input (b) in response to errors in the initial conditions of the state estimators.

is shown in Figure $7 \mathrm{~b}$. Both states were given initial values equal to $90 \%$ of their true values. The same controller gains were used. These simulations show the ability of the minimumphase output predictor structure to reject disturbances and errors in the initial conditions of the state estimator and to track set point changes for a nonlinear, nonminimum-phase system.

\section{Conclusions}

A complete control structure for nonlinear systems with unstable zero dynamics has been developed. The minimumphase output predictor structure is based on using a statically equivalent, minimum-phase output for the process. A GLC feedback control law is used for the resulting system, which is minimum phase. A corrective signal consisting of the difference between the minimum-phase output and the nonminimum-phase output, based on state estimates, is added to the measured output signal, and the result is compared to the set point value with the error fed to the GLC controller. This completes the control structure. The choice of statically equivalent, minimum-phase output is completely arbitrary when defining the control structure, although the performance characteristics will depend strongly on this choice. The notion of 
ISE-optimal output for a nonlinear system was defined, and necessary conditions for ISE optimality for a limited class of nonlinear systems were given. Computer simulations for a chemical reactor system show the ability of the control structure to reject disturbances, errors in the initial conditions of the state estimator, and to track set point changes.

In this work, analytical ISE optimality results have been derived for a limited class of nonlinear systems. Future work will address the problem of numerical calculation of the ISEoptimal output; the minimum-phase output predictor structure will then apply unchanged.

\section{Acknowledgment}

Financial support from Dow Chemical USA is gratefully acknowledged. The authors wish to thank Nikolaos Kazantzis for his valuable comments and criticism.

\section{Notation}

Adj $M=$ adjugate of the matrix $M$

$a d_{f, g}^{k}=k$ th Lie bracket of $f$ and $g$

$A, b, c=$ matrices in standard state space description of a linear system

$c^{*}=$ row vector that defines the minimum-phase output for a linear system

$c_{A}=$ concentration of $A$ in the effluent stream

$c_{A O}=$ inlet concentration of $A$

$c_{B}=$ concentration of $B$ in the effluent stream

$\operatorname{det} M=$ determinant of the matrix $M$

$F=$ flow rate through CSTR

$F(s)=$ desired transfer function between minimum-phase output and set point for a linear system

$f(x), g(x)=$ vector fields that characterize the state model of a nonlinear process

$G_{c}(s)=$ controller transfer function

$G_{p}(s)=$ process transfer function

$G_{p-}(s)=$ minimum-phase part of factorization of process transfer function

$G_{p+}(s)=$ all-pass part of factorization of process transfer function

$h(x)=$ scalar field that determines the process output map

$h^{*}(x)=$ scalar field that determines the process minimum-phase output map

$k_{i}=$ reaction rate constants in simulation example

$K_{c}=$ external PI controller gain in simulation example

$L_{f}^{k} h(x)=k$ th-order Lie derivative of $h$ with respect to $f$

$n=$ order of system

$q(x)=$ nontrivial solution to set of partial differential equations used in obtaining a natural coordinate representation of a nonlinear system

$r=$ relative order

$r_{i}=$ reaction rate in simulation example

$s=$ Laplace transform variable

$t=$ time

$u=$ manipulated input

$v=$ reference input in ISE criterion and GLC transformed control variable

$V=$ volume of CSTR in simulation example

$x=$ vector of state variables

$y=$ process output

$y^{*}=$ statically equivalent, minimum-phase output

$z=$ state variables of zero dynamics of a nonlinear system

\section{Greek letters}

$\alpha_{i}=$ coefficients of characteristic equation of a linear system $\beta_{k}=$ coefficients of characteristic equation in GLC linearized $v-y$ system

$\epsilon=$ design parameter

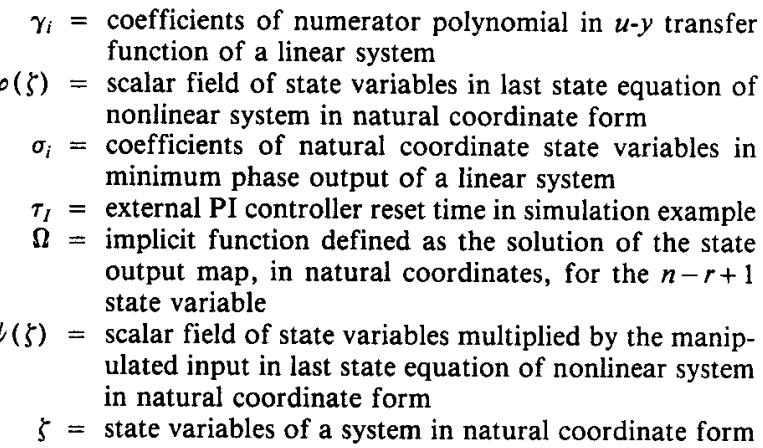

\section{Other symbols}

$\epsilon=$ belongs to

$\boldsymbol{R}=$ real line

$\boldsymbol{R}^{n}=n$-dimensional Euclidean space

\section{Superscripts}

$$
\begin{aligned}
e & =\text { equilibrium value } \\
& =\text { estimated value }
\end{aligned}
$$

\section{Subscripts}

$s=$ steady-state value

$s p=$ set point value

\section{Literature Cited}

Byrnes, C. I., and A. Isidori, "Global Feedback Stabilization of Nonlinear Systems," Proc. IEEE CDC, 1031, Ft. Lauderdale, FL (1985).

Economou, C., M. Morari, and B. O. Palsson, "Internal Model Control: 5. Extension to Nonlinear Systems," Ind. Eng. Chem. Proc. Des. Dev., 25, 403 (1986).

Garcia, C. E., and M. Morari, "Internal Model Control: 1. A Unifiying Review and Some New Results," Ind. Eng. Chem. Proc. Des. Dev., 21, 308 (1982).

Gel'fand, I. M., and S. V. Fomin, Calculus of Variations, Prentice Hall, Englewood Cliffs, NJ (1963).

Hirschorn, R. M., "Invertibility of Nonlinear Control Systems," SIAM J. Control Optim., 17, 289 (1979).

Hunt, L. R., R. Su, and G. Meyer, "Global Transformations of Nonlinear Systems," IEEE Trans, Automat. Contr., AC-28, 24 (1983).

Johnson, C. D., "Singular Solutions in Problems of Optimal Control," Adv. in Contr. Sys., 2, 209 (1985).

Johnson, C. D., and J. E. Gibson, "Singular Solutions of Problems in Optimal Control,"' IEEE Trans. Automat. Contr., p. 4 (1963).

Kantor, J. C., "Stability of State Feedback Transformations for Nonlinear Systems-Some Practical Considerations," Proc. ACC, 1014, Seattle (1986).

Kelley, H. J., "A Transformation Approach to Singular Subarcs in Optimal Trajectory and Control Problems," SIAM J. Contr., 2, 234 (1964).

Kravaris, C., "Input/Output Linearization: a Nonlinear Analog of Placing Poles at the Process Zeros," AIChE J., 34, 11, 1803 (1988).

Kravaris, C., and C. B. Chung, "Nonlinear Feedback Synthesis by Global Input/Output Linearization," AIChE J., 33, 592 (1987).

Kravaris, C., and P. Daoutidis, "Nonlinear State Feedback Control of Second-Order Nonminimum-Phase Nonlinear Systems," Comp. and Chem. Eng., 14, 439 (1990).

Newton, G. C., L. A. Gould, and J. F. Kaiser, Analytical Design of Linear Feedback Controls, Wiley, New York (1957).

Nikolaou, M., and V. Manousiouthakis, "Stability Aspects of Exact Linearization Methods: A Hybrid Approach," Proc. Amer. Control Conf., 2736 (1990).

Ramanathan, S., R. L. Curl, and C. Kravaris, "Dynamics and Control of Quasirational Systems," AIChE J., 35, 1017 (1989). 
Smith, C. A., and A. B. Corripio, Principles and Practice of Automatic Process Control, Wiley, New York (1985).

Van de Vusse, J. G., "Plug-Flow-Type Reactor versus Tank Reactor," Chem. Eng. Sci., 19, 994 (1964).

Wonham, W. H., and C. D. Johnson, "Optimal Bang-Bang Control with Quadratic Performance Index," J. Basic Eng., Trans. ASME, 107 (1964).

Wright, R. A., "Equivalent Output Formulations of Nonlinear Control Problems,"' PhD Thesis, Univ. of Michigan, Ann Arbor (1990).

\section{Appendix A: Nonlinear Systems in Natural Coor- dinates}

Consider a nonlinear system of the form of Eq. 29 such that: (i) the vector fields $g(x), a d_{f}^{1} g(x), \ldots, a d_{f}^{n-1} g(x)$ are linearly independent; (ii) the set of vector fields $\left\{g(x), a d_{f}^{1} g(x)\right.$, $\left.\ldots, a d_{f}^{n-2} g(x)\right\}$ is involutive. Then, the system of partial differential equations,

$$
\begin{gathered}
\sum_{i=1}^{n} g_{i}(x) \frac{\partial w}{\partial x_{i}}=0 \\
\sum_{i=1}^{n}\left[a d_{f}^{1} g\right]_{i}(x) \frac{\partial w}{\partial x_{i}}=0 \\
\vdots \\
\sum_{i=1}^{n}\left[a d_{f}^{n-2} g\right]_{i}(x) \frac{\partial w}{\partial x_{i}}=0
\end{gathered}
$$

admits a nontrivial solution $w=q(x)$ and the transformation,

$$
\zeta=T(x)=\left[\begin{array}{c}
q(x) \\
L_{f} q(x) \\
\vdots \\
L_{f}^{n-1} q(x)
\end{array}\right]
$$

is invertible and transforms Eq. 29 into natural coordinates (Hunt et al., 1983):

$$
\begin{aligned}
\dot{\zeta}_{1} & =\zeta_{2} \\
\dot{\zeta}_{2} & =\zeta_{3} \\
\vdots & \\
\dot{\zeta}_{n-1} & =\zeta_{n} \\
\dot{\zeta}_{n} & =\varphi(\zeta)+\psi(\zeta) u \\
y & =h(\zeta)
\end{aligned}
$$

where

$$
\begin{aligned}
\varphi & =\left[L_{f}^{n} q(x)\right]_{T^{-1}(\zeta)} \\
\cdot \quad \psi & =\left[L_{g} L_{f}^{n-1} q(x)\right]_{T^{-1}(\zeta)}
\end{aligned}
$$

If the system of Eq. 29 has relative order $r$, this easily translates into

$$
\begin{aligned}
& \frac{\partial h}{\partial \zeta_{n-k}}(\zeta)=0, \text { for } k=0, \ldots r-2 \\
& \frac{\partial h}{\partial \zeta_{n-r+1}}(\zeta) \neq 0 .
\end{aligned}
$$

Consequently, the system may be more precisely represented in natural coordinates as Eq. 36.

\section{Proposition $\boldsymbol{A 1}$}

The local asymptotic stability characteristics of the zero dynamics of the system of Eq. 36 depend on the location of the roots of the polynomial in $\xi$ :

$$
\begin{aligned}
\mathbf{P}(\xi ; \zeta)=\frac{\partial h}{\partial \zeta_{1}}(\zeta)+\frac{\partial h}{\partial \zeta_{2}}(\zeta) \xi+\ldots \\
+\frac{\partial h}{\partial \zeta_{n-r}}(\zeta) \xi^{n-r-1}+\frac{\partial h}{\partial \zeta_{n-r+1}}(\zeta) \xi^{n-r}
\end{aligned}
$$

In particular, the zero dynamics of the system of Eq. 36 will be locally asymptotically-stable if and only if the roots of the polynomial (Eq. A4) are in the left half-plane in a neighborhood of the equilibrium point.

\section{Proof}

Applying the general construction in Byrnes and Isidori (1985), the coordinate transformation

$$
z_{1}=\zeta_{1}
$$

$$
\begin{aligned}
z_{n-r} & =\zeta_{n-r} \\
z_{n-r+1} & =h\left(\zeta_{1}, \ldots, \zeta_{n-r}, \zeta_{n-r+1}\right) \\
z_{n-r+2} & =L_{f} h\left(\zeta_{3}, \ldots, \zeta_{n-r}, \zeta_{n-r+1}\right) \\
& =\zeta_{2} \frac{\partial h}{\partial \zeta_{1}}(\zeta)+\ldots+\zeta_{n-r+1} \frac{\partial h}{\partial \zeta_{n-r}}(\zeta)+\zeta_{n-r+2} \frac{\partial h}{\partial \zeta_{n-r+1}}(\zeta) \\
& \vdots \\
z_{n} & =L_{f}^{r-1} h\left(\zeta_{1}, \ldots, \zeta_{n-r}, \zeta_{n-r+1}\right)
\end{aligned}
$$

defined locally around an equilibrium point of the system of Eq. 36 transforms it into

$$
\begin{aligned}
& \dot{z}_{1}= z_{2} \\
& \vdots \\
& \dot{z}_{n-r-1}= \\
& \dot{z}_{n-r}= \\
& \dot{z}_{n-r+1}=z_{n-r+2}\left(z_{1}, \ldots, z_{n-r}, y\right) \\
& \vdots \\
& \dot{z}_{n}=\hat{\varphi}(z)+\hat{\psi}(z) u \\
& y=
\end{aligned}
$$


where

$$
\zeta_{n-r+1}=\Omega\left(\zeta_{1}, \ldots, \zeta_{n-r}, y\right)
$$

is the implicit function defined as the solution of:

$$
h\left(\zeta_{1}, \ldots, \zeta_{n-r}, \zeta_{n-r+1}\right)=y
$$

Consequently, the zero dynamics of the system of Eq. 36 is given by:

$$
\begin{aligned}
& \dot{z}_{1}=z_{2} \\
& \vdots \\
& \dot{z}_{n-r-1}=z_{n-r} \\
& \dot{z}_{n-r}=\Omega\left(z_{1}, \ldots, z_{n-r}, y\right)
\end{aligned}
$$

From the form of the zero dynamics (Eq. A9), it can immediately be concluded that the local internal stability characteristics of the system of Eq. A9 depend on the location of the roots of the polynomial in $\xi$ :

$$
\frac{\partial \Omega}{\partial z_{1}}-\frac{\partial \Omega}{\partial z_{2}} \xi-\ldots-\frac{\partial \Omega}{\partial z_{n-r}} \xi^{n-r-1}+\xi^{n-r}
$$

Due to how $\Omega$ was defined, the partial derivatives of $\Omega$ can be obtained from the implicit function theorem:

$$
\frac{\partial \Omega}{\partial z_{i}}=-\frac{\frac{\partial h}{\partial \zeta_{i}}}{\frac{\partial h}{\partial \zeta_{n-r+1}}}, i=1, \ldots, n-r
$$

Hence, the result.

\section{Corollary}

If $r=n$, the system (Eq. 36) is always minimum phase. If $r=n-1$, the system (Eq. 36) will be locally minimum phase if and only if

$$
\frac{\frac{\partial h}{\partial \zeta_{1}}(\zeta)}{\frac{\partial h}{\partial \zeta_{2}}(\zeta)}>0
$$

in a neighborhood of the equilibrium point. If $r=n-2$, the system (Eq. 36) will be locally minimum phase if and only if

$$
\frac{\partial h}{\partial \zeta_{1}}(\zeta), \frac{\partial h}{\partial \zeta_{2}}(\zeta), \text { and } \frac{\partial h}{\partial \zeta_{3}}(\zeta)
$$

have the same sign in a neighborhood of the equilibrium point. In general, the system (Eq. 36 ) will be locally minimum phase if and only if the first column of the Routh array of the polynomial of Eq. A4 has all its elements positive in a neighborhood of the equilibrium point.

\section{Remark $A 1$}

Note the similarity of the result of Proposition 1 to the linear case. The state space realization given by Eq. 12 is the linear analog of $\mathrm{Eq} .36$, and the zeros of the system of Eq. 12 are the roots of

$$
\gamma_{1}+\gamma_{2} \xi+\ldots+\gamma_{n-r+1} \xi^{n-r}
$$

which is the linear analog of Eq. A4.

\section{Appendix B: Proof of Theorems and Corollary Proof of Theorem 1}

To prove (a), observe that for every final equilibrium point $\zeta^{e}=\left(\zeta_{1}(\infty), 0, \ldots, 0\right)$,

$$
h\left(\zeta_{1}(\infty), 0, \ldots, 0\right)=v
$$

which is one of the terminal conditions of the ISE-optimal trajectories. On the other hand, $\Omega^{*}$ must satisfy

$$
\Omega^{*}\left(\zeta_{1}(\infty), 0, \ldots, 0, v\right)=0
$$

for every equilibrium point. Given the definition of $\Omega^{*}, h^{*}$ must then satisfy:

$$
h^{*}\left(\zeta_{1}(\infty), 0, \ldots, 0\right)=v
$$

To prove (b), observe that from the definition of an ISEoptimal output, the ISE-optimal trajectories can be calculated by solving the set of ODE's:

$$
\begin{aligned}
& \dot{\zeta}_{1}=\zeta_{2} \\
& \dot{\zeta}_{2}=\zeta_{3}
\end{aligned}
$$

$$
\dot{\zeta}_{n-r}=\Omega^{*}\left(\zeta_{1}, \ldots, \zeta_{n-r}, v\right)
$$

with the given set of initial conditions and value of $v$. Since the function $h^{*}$ (and therefore $\Omega^{*}$ ) is independent of the initial conditions $\zeta_{1, o}, \ldots, \zeta_{n-r, o}$, this means that the response of Eq. B3 must satisfy $h\left(\zeta_{1}(\infty), 0, \ldots, 0\right)=v, \zeta_{2}(\infty)=0, \ldots$, $\zeta_{n-r}(\infty)=0$ for every set of initial conditions. In other words, the system of Eq. B3 is asymptotically stable. On the other hand, calculating the zero dynamics of the system of Eq. 39 (see Proposition Al of Appendix A) yields:

$$
\begin{aligned}
\dot{z}_{1} & =z_{2} \\
& \vdots \\
\dot{z}_{n-r-1} & =z_{n-r} \\
\dot{z}_{n-r} & =\Omega^{*}\left(z_{1}, \ldots, z_{n-r}, y^{*}\right)
\end{aligned}
$$

Comparing Eqs. B3 and B4, it is observed that they represent exactly the same system. Hence, the system of Eq. B4 is internally asymptotically stable, that is, the system of Eq. 41 is minimum phase. 


\section{Proof of Theorem 2}

Due to the special form of the dynamics, the ISE optimization problem is a calculus of variations problem:

Minimize

$$
\mathrm{ISE}=\frac{1}{2} \int_{0}^{\infty}\left[v-h\left(\zeta_{1}, \frac{d \zeta_{1}}{d t}, \ldots, \frac{d^{n-r} \zeta_{1}}{d t^{n-r}}\right)\right]^{2} d t
$$

subject to the boundary conditions

$$
\begin{array}{cc}
\zeta_{1}(0)=\zeta_{1, o} & h\left(\zeta_{1}(\infty), 0, \ldots, 0\right)=v \\
\frac{d \zeta_{1}}{d t}(0)=\zeta_{2, o} & \frac{d \zeta_{1}}{d t}(\infty)=0 \\
\vdots & \vdots \\
\frac{d^{n-r+1} \zeta_{1}}{d t^{n-r+1}}(0)=\zeta_{n-r, o} & \frac{d^{n-r+1} \zeta_{1}}{d t^{n-r+1}}(\infty)=0
\end{array}
$$

Hence, the optimal trajectories must satisfy the Euler-Lagrange equation, which for the above performance index is given by Eq. 40 .

\section{Proof of corollary}

a) The expression,

$$
h\left(\zeta_{1}, \frac{d \zeta_{1}}{d t}, \ldots, \frac{d^{n-r} \zeta_{1}}{d t^{n-r}}\right)=v
$$

is a trivial $(n-r)$ th integral of Eq. 40 . Furthermore, when the above expression is viewed as a differential equation in $\zeta_{1}$, driven by arbitrary initial conditions, its solution always satisfies the terminal conditions (Eq. 41), as an immediate consequence of the minimum-phaseness of $h$. Hence, the choice $h^{*}=h$ satisfies the necessary conditions for optimality. Finally, it is clear that perfect control of $h^{*}$ to $v$ leads to ISE $=0$.

b) For a system of relative order $r=n-1$, the Euler-Lagrange equation (Eq. 40) becomes:

$$
\begin{aligned}
\frac{\partial h}{\partial \zeta_{1}}\left(\zeta_{1}, \frac{d \zeta_{1}}{d t}\right) & {\left[v-h\left(\zeta_{1}, \frac{d \zeta_{1}}{d t}\right)\right] } \\
- & \frac{d}{d t}\left\{\frac{\partial h}{\partial \zeta_{2}}\left(\zeta_{1}, \frac{d \zeta_{1}}{d t}\right)\left[v-h\left(\zeta_{1}, \frac{d \zeta_{1}}{d t}\right)\right]\right\}=0 .
\end{aligned}
$$

The trivial first integral,

$$
v-h\left(\zeta_{1}, \frac{d \zeta_{1}}{d t}\right)=0
$$

fails to satisfy the terminal condition

$$
h\left(\zeta_{1}(\infty), 0\right)=v
$$

because the resulting trajectory $\zeta_{1}(t)$ is unstable due to the nonminimum-phaseness of $h$. Using a standard result in the theory of calculus of variations (Gel'fand and Fomin, 1963) for the second-order Euler-Lagrange equation, it can easily be seen that the entire family of first integrals of Eq. B6 is given by

$$
\begin{aligned}
& \frac{1}{2}\left(v-h\left(\zeta_{1}, \frac{d \zeta_{1}}{d t}\right)\right)^{2} \\
& +\frac{d \zeta_{1}}{d t} \frac{\partial h}{\partial \zeta_{2}}\left(\zeta_{1}, \frac{d \zeta_{1}}{d t}\right)\left[v-h\left(\zeta_{1}, \frac{d \zeta_{1}}{d t}\right)\right]=C
\end{aligned}
$$

where $C$ is an arbitrary constant. To satisfy the terminal time condition, $h\left(\zeta_{1}(\infty), 0\right)=v$, we must choose $C=0$. Hence,

$$
\begin{aligned}
& \frac{1}{2}\left[v-h\left(\zeta_{1}, \frac{d \zeta_{1}}{d t}\right)\right]^{2} \\
& +\frac{d \zeta_{1}}{d t} \frac{\partial h}{\partial \zeta_{2}}\left(\zeta_{1}, \frac{d \zeta_{1}}{d t}\right)\left[v-h\left(\zeta_{1}, \frac{d \zeta_{1}}{d t}\right)\right]=0
\end{aligned}
$$

or equivalently

$$
h\left(\zeta_{1}, \frac{d \zeta_{1}}{d t}\right)+2 \frac{d \zeta_{1}}{d t} \frac{\partial h}{\partial \zeta_{2}}\left(\zeta_{1}, \frac{d \zeta_{1}}{d t}\right)=v
$$

is the required (nontrivial) first integral. Since the ISE-optimal output, $h^{*}$, must match the above integral, which is unique, we will have:

$$
h^{*}\left(\zeta_{1}, \frac{d \zeta_{1}}{d t}\right)=h\left(\zeta_{1}, \frac{d \zeta_{1}}{d t}\right)-2 \frac{d \zeta_{1}}{d t} \frac{\partial h}{\partial \zeta_{2}}\left(\zeta_{1}, \frac{d \zeta_{1}}{d t}\right)
$$

Hence, the result.

Manuscript received Feb. I2, 1991, and revision received Oct. 14, 1991. 